\title{
What is a virtual world? Definition and classification
}

\author{
Carina Girvan ${ }^{1}$ (D)
}

Published online: 8 February 2018

(C) The Author(s) 2018. This article is an open access publication

\begin{abstract}
In 2008, articles by Bell and Schroeder provided an initial platform from which to develop a coherent definition of the term 'virtual worlds'. Yet over the past ten years, there has been little development of the term. Instead there is confusion in the literature, with the introduction of new terms which are at times used to classify the type of virtual world and at others are used synonymously with the term. At the same time there has been a resurgence of interest in the potential of virtual reality which further muddies the conceptual waters. While the lack of a clear and common understanding of a term is not uncommon, there are implications for researchers and practitioners. To address these issues, this paper presents a new framework for the definition of virtual worlds, arguing what it is for a world to be virtual, the user experience that is a necessary part of this and the technical features which afford this. For the first time the relationships between commonly confused terms and technologies are identified to provide a much needed conceptual clarity for researchers and educators.
\end{abstract}

Keywords Virtual world $\cdot$ MUVE $\cdot 3 \mathrm{D}$ learning environment $\cdot$ Definition

\section{Introduction}

Thus far, definitions of virtual worlds lack an essential conceptualisation of what a virtual world is. The propensity towards a techno-centric definition has its advantages as it allows for a myriad of user experiences, however it results in confusion between technologies with similar technical features, most likely because a virtual world, much like a smart phone, relies on a combination of different technologies. For example, it is unclear how Bell's (2008) definition of a virtual world could not as easily be applied to a MMORPG, whilst at the same time others argue that virtual worlds are not games. However, solely focusing on

Carina Girvan

girvanc@cardiff.ac.uk

1 School of Social Sciences, Cardiff University, Cardiff, Wales, UK 
the experience is equally insufficient as we might consider that such definitions could equally be used to describe the experience of learners using a range of collaborative eLearning tools.

The inconsistent use of terms and descriptors means that it is unclear as to whether these terms are synonymous or refer to subtle differences between applications. It also raises questions as to whether research conducted in one is relevant to the others and to what extent there is a natural overlap. This inconsistency is not uncommon in the field of educational technology, but as Oliver (2005) notes, a concept that is allowed to drift and become ambiguous, risks becoming analytically worthless. While it is important to accurately define what is meant by virtual world in order to guide research in the area (Schroeder 2008), it is equally important for educational practice and policy making. For instance, practitioners learning about the potential of virtual worlds for education may be unable to provide the experiences for their learners that they had expected, due to investment in technologies incorrectly labelled as virtual worlds. As a result, definitions have implications from the purchasing of technology in a school, to the expectations we have around its use.

Any technology is an artefact within a wider milieu. Therefore, a definition of a technology needs to take into account the activities and practices when using it, as well as the social arrangements and organisational forms surrounding its use. Each time we reengage with a technology as part of our everyday social experience, we encounter it afresh, re-establishing and reformulating its use in terms of both function and functionality. Thus a definition needs to encompass these many possibilities and be sufficiently flexible to future developments, whilst maintaining its distinctiveness from other technologies.

To achieve a clear and usable definition of a virtual world, this article begins by examining the current literature, highlighting the importance of the user experience within the rationales given for using virtual worlds in education, whilst popular definitions such as Bell's focus on the technical aspects of the technology. While there are often subtle differences in definition or description of virtual worlds between articles, other terms such as multi-user virtual environments (MUVEs) or 3D immersive virtual environments, have had varying popularity and can be considered to be either bridging terms, used to draw in a reader's familiarity with existing concepts to bridge a gap in experience, or delineating terms, to signal the uniqueness of the technology. Yet problems emerge when different terms are used to refer to the same technology, or when a single term is used to refer to disparate things resulting in the term becoming conceptually devalued and introducing confusion.

We then take a step back to conceptualise what a world is and what it means for this world to be virtual in relation to the experience of its users and the necessary underpinning technical features. This takes into account that as features and uses can change over time, any definition must balance specificity and flexibility for it to have long-term value. To complete the definition, the relationship between virtual worlds and other, seemingly similar terms and intersecting technologies are explored.

While this paper predominantly draws on research from education, the definition and implications are of relevance to anyone interested in using or conducting research using virtual worlds. 


\section{Background: competing terms and the complexity within}

The development of virtual worlds, both in terms of technical features and the widening range of reported user experiences, has resulted in a fragmented understanding in the literature of what a virtual world is and is not. To further complicate this problem, within the educational research literature we find a variety of terms which are used to label the technology: virtual world (VW); virtual environment (VE); multi-user virtual environment (MUVE); massively-multiplayer online (role-playing) game (MMO(RP)G); immersive virtual world (IVW); immersive world; immersive online environment; 3D virtual learning environment; open-ended virtual worlds; simulated worlds; serious virtual world; social virtual world; synthetic virtual world; and virtual learning environment (VLE). This seemingly innocuous plethora of terms has the potential to introduce confusion through conceptual misunderstanding, with terms operating as buzzwords or fuzzwords, limiting the development of the field and its impact on education. This section examines the ways in which these terms have been used and defined.

As noted, a variety of terms are currently used across the literature but often without conceptual clarity. It is not uncommon for articles to use more than one term to describe a single application (e.g. Richard and Taylor 2015). MUVE and virtual world are both used to label Second Life in one article by Honey et al. (2012), while Ghanbarzadeh et al. (2014) consider virtual worlds to be a subset of MUVEs. Others have labelled Second Life an immersive virtual world (McArdle and Bertolotto 2012), a social virtual world (Jarmon and Sanchez 2008), and a virtual environment (Singh and Lee 2009), yet virtual environment is also used by Minocha et al. (2010) to label Facebook. Reasons for exploring the use of virtual worlds in education are often associated with the technical features of the technology and the opportunities they afford. However, it is the educational activities created by the teachers/researchers and the engagement of learners and teachers in these activities within the virtual world that bring about the educational experience and outcomes. As a result, 'serious' or 'educational' are often unhelpful prefixes to 'virtual world', particularly when referring to applications such as Second Life which afford a wide range of user experiences. This hints at an implicit categorisation of virtual worlds in relation to other technologies, however this is often not made explicit, nor used consistently within the field.

Duncan et al. (2012) provide a taxonomy of virtual world usage in education. This is based on an over-extended use of the term virtual world to "encompass any online environment that allows users to play, learn or interact" (p. 950) and issues emerge with the distinctions initially drawn between MUVEs, virtual worlds and virtual learning environments (VLEs). Virtual worlds are described as encompassing MUVEs with World of Warcraft, Second Life and Active Worlds initially classified as MUVEs, although later Second Life and Active Worlds are also classified as VLEs within the sub-category of 3D web technologies. Learning management systems (LMSs) such as Blackboard and Moodle are also classified as a sub-category of VLEs. It therefore appears that the authors consider MUVEs and virtual worlds to be sub-categories of VLEs. However, there is ongoing confusion as the article refers to all of these applications as virtual worlds.

Calling a virtual world a VLE is problematic, not least because LMSs are commonly referred to as VLEs in the wider educational research literature. Stone (2013) highlights virtual worlds have several features and characteristics which are not found in VLEs and so could not be a sub-category of VLE. Further, Kemp et al. (2009) developed a system for the integration of the VLE Moodle with Second Life known as SLOODLE. As we have established, virtual worlds such as Second Life, Active Worlds and Minecraft do not only 
provide opportunities for educational activities to occur but can be appropriated for such purposes, however VLE/LMSs are designed for the purpose of education. The research literature on VLEs has limited relevance to educational research in virtual worlds beyond that of any eLearning or distance education literature. Similarly, institutional policies on the use of VLE/LMSs do not easily translate into virtual world technologies. What is clear from this is that VLEs and virtual worlds are distinct technologies with some technical overlap but are designed to provide quite distinct user experiences and while a virtual world is not a VLE, a VLE can be created in a virtual world.

This brings us to the definitional complexity and inconsistency of terms which may explain the inconsistent use of terms explored above. By far the most commonly used definition of virtual world in the current literature is Bell's, cited over 500 times:

A synchronous, persistent network of people, represented as avatars, facilitated by networked computers (Bell 2008, p. 2)

It combines elements of earlier definitions by Bartle (2004) and Castronova (2005) to address the lack of a commonly used and accepted definition of virtual worlds, and was presented with the intention of starting a discourse on defining virtual worlds.

The key component of this definition which sets virtual worlds apart from other technologies is the avatar. Bell makes a clear distinction between avatars used to represent a user in an application such as Facebook, in which the avatar is a static representation of the user on a website, and avatars in virtual worlds, in which the avatar has agency. However, there is no clarification on whether multiplayer games, which have all of the features mentioned within the definition are virtual worlds or not.

While this is the most commonly used definition, many authors offer a description of virtual worlds or add to this definition in a number of ways, but again we see inconsistency across the literature even when the same applications are being described. For example, Webber (2013) is one of the few authors to highlight an economy as a feature of Second Life which has its own currency with fluctuating exchange rate against the US dollar and can be used to purchase goods and services in-world. Yet, not all virtual worlds have a discrete economy which users have to engage with in order to participate in the virtual world, so while useful in the description of Second Life it is not broadly applicable to virtual worlds. Allison et al. (2012) emphasise the importance of users creating their own environment and not being constrained by what Gee (2003) calls 'game-grammar'; while Sclater and Lally (2013) use this to distinguish between virtual worlds which are and are not game-based, using the term 'open-ended virtual worlds' for those which are not gamebased. The issue as to whether or not the virtual world of Second Life, for example, is or is not a game has been raised regularly in the past (for example Grassian and Trueman 2007; Bainbridge 2007) and continues in conference discussions and within classrooms today. It also raises issues around the inclusion or exclusion of MMO(RP)Gs such as World of Warcraft, in any definition of virtual worlds.

It is not uncommon for definitions and descriptions of virtual worlds to focus on technical features, but the rationale for the use of virtual worlds in education is often linked to the types of activities that the combination of features affords. This highlights the importance of the user experience over the technical, and by way of contrast to the more popular techno-centric definitions, Schroeder's less cited definition (published alongside Bell's) positions virtual worlds as a subset of virtual environments, distinguishing them as virtual environments which provide a particular form of user experience: 
persistent virtual environments in which people experience others as being there with them - and where they can interact with them (Schroeder 2008, p. 2).

The social experience is afforded primary importance by Schroeder and he asserts that this is what distinguishes virtual worlds from MMORPGs. Educational researchers often refer to the opportunities for social constructivist learning activities as a rationale for the use of virtual worlds. However, there are a range of user experiences that are referred to in the literature and a focus on the experience of the user can be as problematic as a technical focus, as it is subjective, depends on a number of factors and tends to be described using terms which are ill-defined themselves, such as 'immersive'.

As the features and user experiences offered by these technologies develops, it is important to have a definition of virtual world which is not reliant on these but instead provides a framework to understand what is necessary and possible. To do this we need to take a step back from specific applications and consider what it means for a world to be considered virtual, the experiences that would be an essential part of this and the technical features that the user would need to engage with to experience this.

\section{Defining virtual worlds}

\section{What makes a virtual world? A conceptualisation}

The term virtual world requires a clear understanding of what is meant by world and what it means to have a world which is virtual.

\section{World}

There are numerous schools of thought on what a world is, including everything that constitutes the physical universe. This is obviously too broad for our purposes, and also ignores the experience of being in a world.

Drawing on Heidegger's concept of world disclosure, we understand the world based on our day-to-day experience of the world, as limited by our world view, whether that is through interactions with others or with objects. Language is an important part of this experience and it is how we come to find meaning in the world, through communication with others. Much of this understanding is pre-interpreted by others and it is their language that we are introduced to and in turn use to describe the world. Others are therefore an important part of any world, which is inhabited and shaped by its inhabitants.

Along with language, our bodies are important to understanding and interpreting our world. "We know ourselves and our world mainly because we live and move in the world through our bodies" (Nguyen and Alexander 1996, p. 117). Some form of embodiment is therefore important in any world and our bodies are likely to shape our experience of that world. How we see, hear or move within the world will inform our understanding of that world. Consider the experience of an adult who is $5 \mathrm{ft}$ tall versus that of someone who is $6 \mathrm{ft}$ tall. Their visual perception of the world is different, their actions (for example, to reach something on a high shelf) are different and their understanding of power relationships may also be different; their bodies shape their understanding of the world. Therefore our embodied navigation of the world, interaction with objects and interactions with others is important. It is through our embodiment, movement and perception within a space, that 
serendipitous encounters can occur and that we can move around an object to gain another perspective on it.

Together with spatial dimensions, there are is also a temporal dimension in any world. Unlike space which is comprised of three dimensions that we can move freely between and at varying speeds, there is only one temporal dimension which moves in one direction and at a consistent pace (although it may be perceived to occur at different rates for different people), preventing us from returning to the same moment in time.

Thus the definition of a world comprises three key ideas: (1) A shared space which is inhabited and shaped by its inhabitants. (2) Experiences and interpretation of those experiences are not fixed but mediated through our physical bodies and psychological responses. (3) Through our physical bodies we move about the shared space, interacting with objects and others, with whom we construct a shared understanding of the world at that time.

\section{Virtual}

Virtual is a modifier to world and therefore it is important to be clear about what is meant by a world which is virtual. Like world, virtuality is also interpreted in many forms, including an aspect of reality which is both ideal and real, or as if it were real. Deleuze (1990) conceptualises the virtual as that which is opposed to actual, but not opposed to real, meaning that the virtual is real, or at least our perception of it is. This highlights a trap which many scholars fall into, describing virtual worlds as "not real". However, placing the virtual and the actual as opposites is also problematic, as actual is often used to describe a reality, existing at the current time. It is argued that the use of real and virtual creates a dichotomous perspective in current research resulting in inaccurate assumptions, for example between virtual identity and real identity, (Lehdonvirta 2010). Therefore, it is perhaps better to contrast the virtual world with the physical world, natural world or material world (Shields 2003) whilst being cognisant that people do not hold one 'real' identity and instead have multiple identities depending on the people they are with and the places that they are in.

Beyond comparing the virtual to the real, physical, natural or material, in the area of technology enhanced learning, virtual is used to describe a simulated experience. This gives a sense of something that is almost real, something which is perceived to exist, but lacks physical properties beyond the screen.

As pre-interpretation shapes our understanding of the world, so too it shapes our understanding of seemingly familiar objects and activities in a virtual world. When we first experience an object we learn about it in relation to a specific context, however we soon learn that this object may appear in other contexts and have other meanings in relation to other activities. It is therefore reasonable to assume that a virtual world, which is a human construct and thus limited by our knowing, is another context or world in which we transfer our knowledge of given objects and their use, however those objects may take on alternative meanings in this alternative world as it represents a cultural space. Together with the development of haptic feedback which provides a physical sensation of an experience in the virtual world, this highlights what Sheilds (1996) describes as the "crisis of boundaries" (p. 7), the interface between the physical or material world and a world which is virtual. While our understanding of an object in the virtual world may be pre-interpreted based on our understanding of that same object in the physical world, we can reconceptualise our understanding within the virtual world with others in that same space, and at the same time with others in the physical world. 
Therefore a virtual world is a simulated environment which meets the framework of a world presented above. However, what makes it distinct from the material or physical world are the types of experience available for the user afforded by the combination of different technical features, most notably the avatar.

\section{Virtual world user experiences}

Using the framework of what a virtual world is, presented above, this section considers two aspects of the user experience which are necessary components of a virtual world: a sense of presence and shaping the world. While these are broad experiences, not related to any specific educational context, they can be applied to such virtual or blended learning contexts equally and highlight key experiential differences between physical and virtual worlds.

\section{Sense of presence}

A user's sense of presence, a sense of being 'in' a virtual world, is an essential user experience which relies on a number of features and may be related to other experiences within the virtual world. Another commonly referred to feature is immersion, but I argue that presence is a more useful term for our purposes. The blurring of boundaries between worlds is particularly important here. Can we feel as though we are in a virtual world and at the same time know that we are in the physical world?

A sense of presence (a feeling of being in a shared space) or a sense of co-presence (being in a shared space with others and with whom the user can interact) is often referred to in the literature (e.g. Boughzala et al. 2012) and is regularly cited as one reason for exploring virtual worlds for educational purposes. A sense of presence suggests that the user feels that they are represented in the space and that others can see that representation of the user. They are aware that others know of their arrival, departure and any of their actions whilst in the virtual world. It is difficult to hide from others within a virtual world, although it is possible to be alone.

Another experience, strongly associated with the sense of presence, is immersion. Although immersion is also often cited as one of the reasons for using virtual worlds in education (e.g. Grenfell and Warren 2010), and perhaps more often than a sense of presence, it is less clear what is understood by immersion. Brown and Cairns (2004) suggest that total immersion results in a loss of awareness of the physical world. However, this is rarely experienced, and users are more likely to experience engagement or engrossment which Brown and Cairns suggest are closely linked to the users' familiarity with the interface and controls.

But what of the learner who does not report any sense of immersion, or categorically states that they do not experience immersion, or those who are new to the interface and controls. Can they still feel present in the virtual world? I would argue that they can, particularly when in the virtual presence of others, through a sense of being co-present in a space with another. It would also be logical that co-located learners (in both physical and virtual worlds), communicating with each other could switch seamlessly between the two spaces in terms of their engagement or engrossment whilst maintaining a sense of presence in both the physical and virtual world. Whilst the level of immersion a user experiences is most likely linked to additional factors, such as the learning experience students are engaged in and their familiarity with the user interface, these are factors which are beyond the virtual world itself. Therefore, whilst immersion is a useful concept, it does not 
encapsulate the range of user experiences that a sense of presence does. Thus, a sense of presence is an essential component whilst immersion is a product, in part, of that experience.

\section{Shaped by its inhabitants}

The user, or inhabitant, of a virtual world becomes both a consumer of content and producer of content. Fisher (2010) describes this type of user as a prosumer, one who is facilitated by a networked infrastructure of tools to create content for others to consume. While this would be common for users of web2.0 tools, such as bloggers and vloggers; I argue that by being an inhabitant of a virtual world, facilitated by embodiment through an avatar, means that users do not only create content for others but also shape the experience of others in that same world. By engaging with the technology-creating an avatar, interacting with others, creating objects, or simply by moving about the world-they are shaping the virtual world in real-time. It could be argued that this is simply the production of an experience that is consumed by others, but those others are also shaping that experience in the same moment. However without the avatar we are unaware of others and they are unaware of us. Thus inhabitant is a much more efficient description of the user, embodied as an avatar.

It is the action or response to actions that shapes the virtual world, as it would in the material world, and is therefore a central experience. How the virtual world is shaped, how it is experienced and the types of experiences (for education or not) that are created therefore, rely on users and the parameters of technical features.

This also emphasises the social nature of virtual worlds, although, just as in the physical world, it is possible for inhabitants to create private spaces for the individual connected to the public or semi-private spaces of the world. This is facilitated through a range of technical features which may vary from virtual world to virtual world. However, a private server for the individual user, I suggest, provides a simulated environment and not a virtual world as there are no other inhabitants within that space.

\section{Virtual world technical features}

The features of individual virtual worlds influence the experience of users within those spaces. Although we can expect the parameters to vary, beyond a simulated environment, the following broad features are required to achieve the experiences described above and meet the requirements of the framework:

\section{Avatars}

Any world is experienced and mediated through our bodies. Within a virtual world this is achieved through the use of an avatar - the inhabitant of the virtual world — which provides the user with an active agent with which to encounter the world. These avatars, their appearance and abilities, mediate our experience of the virtual world and our interactions with others. While the user could render their avatar as invisible, the presence of each avatar is registered within the system, making their actions observable. By affording embodied movement within the virtual world, avatars support our sense of presence within a shared space. 
The avatar provides the body through which the user experiences the virtual world, moving in three dimensions, interacting with objects and others, within the parameters of that virtual body and the input device or the user's skill in using that device to control the avatar. Thus, the user experiences the virtual world through the avatar, but that experience is not necessarily limited to that avatar's view. In a first-person view we do not see the avatar represented but others see our avatar and its actions, a similar experience to our everyday lives. However, virtual worlds allow us to take a third-person view, which is a typical default view. Thus we see both our own and other's avatars and how they interact within the shared space. The more realistic these actions, or the more closely they resemble the interactions between people in the physical world, the more likely they are to support a sense of being in a space with others (Yee et al. 2007).

\section{Multiple concurrent users}

In order to support a sense of being in a shared space with others, a virtual world has to support multiple users to $\log$ in at the same time. This requires each user to connect to a central server via a client on their computer, via a public or local network. As such each user must experience the passage of time within the virtual world in the same way. This is achieved in real-time. Although we could imagine a virtual world which operates at, for example, double or half the speed of our physical world, our actions within the virtual world would still be constrained by the timing of our actions in the physical world. Therefore, because of the blurred boundary between these two worlds, the passage of time in a virtual world is the same as, or constrained by, the physical world.

\section{Communication tools}

Communication tools are essential in virtual worlds, facilitating communication between users. Depending on the application these tools include voice and text based media, through public and private messaging systems. It could also include the upload of images, symbols or the design of shared space. It is through these communication tools that we coconstruct a shared understanding of the world that we, through our avatars, inhabit.

\section{Content creation tools}

In order to support the creation of content by users, construction and programming features need to be available. This may include tools to upload content created in external software packages, such as 3D meshes in Blender. Some virtual worlds, such as There.com, provide users with the opportunity to create content but this needs to be submitted and approved before it can appear in-world. While this limits the prosumer experience and limits the sense of immersion, more popular virtual worlds such as Active Worlds and Second Life allow users to freely generate and distribute content whilst remaining in the virtual world throughout.

\section{Persistence}

Like any world, inhabitants will come and go. Persistence ensures that some trace of their actions remains. A persistent environment remains whether users are logged in or not, retains the location of people and objects as well as information about object ownership. 
Thus, if a user creates an object and leaves it within the virtual world, a second user will view the object in the same location whether the first user is online or not. If the second user removes the object from the location, when the first user returns to the location they will no longer be able to view the object. This is achieved through the use of a client-server architecture, in which the central server(s) manages the persistence and interactions by storing serialized versions of the objects the user creates, and is an essential feature to enable a prosumer experience.

\section{Representation of space}

Finally the technology must provide users with a graphical representation of the shared space, the user within that space, as well as other users and objects within that space. This is a dynamic representation facilitated through the client-server architecture, showing each action that a user makes within the virtual world.

The extent to which representational fidelity is a factor in a learner's engagement is contested. While Dionisio et al. (2013) suggest that the extent to which the virtual world is realistic may impact the extent to which users feel psychologically and emotionally immersed, we can question whether this would limit engagement or the user's sense of presence within that space, particularly if we consider the popularity of applications such as Minecraft.

\section{Virtual worlds: their relationships to other technologies}

Having identified what is meant by a world that is virtual, the types of experiences and the features of the technology that enable this experience, this section provides a relational framework to explain how virtual worlds intersect with technologies and terms that have previously been used to provide a bridge in understanding when virtual worlds were first entering mainstream consciousness, but at this point add to the confusion in the use of the term virtual world. Following Salen and Zimmermen's (2004) explanation of the complex relationship between the terms play and game, this section presents the relationship between virtual worlds and commonly confused terms. As some of these relationships are apparently bidirectional, for clarity each relationship between terms is presented followed by the rationale for this relationship.

\section{Relationship: a virtual world is a subset of world}

First, it is important to clarify that a virtual world is not dichotomous to the real, physical or material world that we, as humans, inhabit. Instead a virtual world is a subset of world within the physical world. With the ongoing development and integration of haptic devices the boundary between these two worlds will become increasingly blurred, however a virtual world will remain a part of our physical world.

\section{Relationship: a virtual world is a subset of virtual environment}

Virtual environment is one of the mostly commonly used alternative terms for platforms otherwise known as virtual worlds. The similarity in terms draws on our existing conceptual frameworks of what it means for something to be virtual, meaning that virtual 
environment has been a useful bridging term. However they are distinct and I argue that a virtual world is a subset of virtual environment. There are many different types of virtual environments which users can explore such as simulations and whilst these environments might be world-like, they are not inhabited as previously described. Failing to clarify this relationship in the past has contributed to the over extension of the term virtual world, treating it as synonymous to virtual environment.

\section{Relationship: a virtual environment is a component of a virtual world}

Every world contains an environment for its inhabitants. In a virtual world, this is described as the virtual environment.

\section{Relationship: a virtual world is a subset of multi-user virtual environment}

Multi-user virtual environment (MUVE) was, for some time, a popular term used in the literature used synonymously with virtual worlds. It is another bridging term as it qualifies the type of virtual environment - one that has multiple users. However without defining the types of user experience, this is also an easily over extended term which can be applied to $\mathrm{MMO}(\mathrm{RP}) \mathrm{Gs}$ and virtual worlds equally, again highlighting the predominance of technical features over user experience in definitions. Instead both MMO(RP)Gs and virtual worlds are subsets of multi-user virtual environments.

\section{Relationship: a multi-user virtual environment is a component of a virtual world}

As presented in the section on the user experience above, it is necessary for any virtual world to have multiple users. These users do not have to be online at the same time but their presence and actions affect our own experience within the virtual world. Thus a virtual world which was for the sole use of a single user, even one using the same platform as a virtual world (for example OpenSim), would be defined as a virtual environment and treated as a simulation.

\section{Relationship: a virtual world is not a MMO(RP)G}

There has been much discussion about the differences between virtual worlds and MMO(RP)Gs. This has been fuelled by the debate mentioned in the first section of this paper on whether a virtual world is a game. As Allison et al. (2012) highlights, virtual worlds emphasise the importance of users creating their own environment and not being constrained by what Gee (2003) calls 'game-grammar'. It is this game-grammar, or lack thereof, that distinguishes these two technologies.

Additionally, this raises an important point about the use of the term open-ended virtual world. The use of this term supposes that some virtual worlds are not open-ended. Although all virtual worlds, like any technology, operate within constraining parameters, all virtual worlds are, by the defining framework presented above open-ended. Therefore this is an unnecessary term. 


\section{Relationship: a MMO(RP)G can exist within a virtual world}

While a lack of game-grammar distinguishes virtual worlds from MMO(RP)Gs, it is possible for users to create games, including $\mathrm{MMO}(\mathrm{RP}) \mathrm{Gs}$ within the virtual world. While they may become a component of a virtual world, they are not a required element.

\section{Relationship: a virtual world can support serious and educational experiences}

As per the argument above, that virtual worlds can provide a game experience, virtual worlds can also provide a serious (non-game), educational or any other specific experience a user chooses to create within the virtual world. A virtual world can be designed to provide only those elements that the designer/teacher wishes to support, however even a virtual world created for educational purposes can be appropriated for other purposes by its inhabitants.

As explained earlier, a LMS/VLE is not a virtual world, however a virtual world could contain access to, draw content from and send data to a VLE. Therefore, while terms such as serious or educational initially appear to be useful modifiers to describe the user experience in different virtual worlds, they only describe one part of any experience that a user may have in these spaces. Similarly immersive is an unhelpful modifier as, following the earlier discussion, an individual user may have an immersive experience, whilst another does not, although both are in the same virtual world.

\section{Relationship: virtual worlds can be accessed through virtual reality systems}

With the recent resurgence of virtual reality (VR) research which is beginning to enter mainstream K-12 education, although typically limited to Google Cardboard and other low-cost options, it is important to provide a clear distinction between virtual worlds and virtual reality as we are already seeing the blurred use of terms in the literature. I would argue that a VR system is a means to immerse users in a virtual world.

VR is a technical system through which a user or multiple users can experience a simulated environment. One such environment is a virtual world. This is most clearly illustrated by the recently retired development of a Second Life viewer for Oculus Rift. Just like a desktop computer, a VR system allows the user to enter the virtual world as an avatar, acting and interacting with its environment. VR systems provide new ways to experience virtual worlds and limit our perception of a world outside the virtual world by enveloping our senses to an ever increasing extent, potentially enhancing our sense of immersion in the virtual world.

Importantly this experience is very different to a user using a VR system to play a single-person game, for example. Therefore a virtual world is simply one type of virtual environment that a user can engage with through a VR system.

\section{Conclusion}

To date, there have been a range of different terms used to describe the same technologies and the over-extension of terms to dissimilar tools. The ambiguity in the use and understanding of the term virtual world limits advancement of research and has the potential to render the term analytically worthless. Without a clear understanding of what a virtual 
world is and how it is similar and distinct to other technologies they may consider using, teachers are unlikely to invest the time and money required to use the technology for educational purposes.

To address this, this paper has presented a new framework for the definition of virtual worlds identifying what a world is, what it means for that world to be virtual, the types of user experiences and technical features that are necessary, and based on these, how virtual worlds differ from other similar technologies. Distilling the above discussion, we can define virtual worlds as:

Shared, simulated spaces which are inhabited and shaped by their inhabitants who are represented as avatars. These avatars mediate our experience of this space as we move, interact with objects and interact with others, with whom we construct a shared understanding of the world at that time.

Acknowledgements I would like to thank colleagues at the Centre for Research in IT in Education, in the School of Education and School of Computer Science and Statistics at Trinity College Dublin for input on early ideas. Support from colleagues in the School of Social Sciences at Cardiff University has been invaluable in the development of this final paper. Finally I would like to thank the reviewers and editor for their recommendations and positive support.

Open Access This article is distributed under the terms of the Creative Commons Attribution 4.0 International License (http://creativecommons.org/licenses/by/4.0/), which permits unrestricted use, distribution, and reproduction in any medium, provided you give appropriate credit to the original author(s) and the source, provide a link to the Creative Commons license, and indicate if changes were made.

Funding Early work was funded through the 'Embark Initiative' by the Irish Research Council for Science Engineering and Technology.

\section{References}

Allison, C., Miller, A., Oliver, I., Michaelson, R., \& Tiropanis, T. (2012). The Web in education. Computer Networks, 56(18), 3811-3824.

Bainbridge, W. S. (2007). The scientific research potential of virtual worlds. Science, 317(5837), 472-476. Bartle, R. (2004). Designing virtual worlds. Indianapolis, IN: New Riders Publishing.

Bell, M. W. (2008). Towards a definition of "virtual worlds". Journal of Virtual Worlds Research, 1(1)

Boughzala, I., De Vreede, G. J., \& Limayem, M. (2012). Team collaboration in virtual worlds: Editorial to the special issue. Journal of the Association for Information Systems, 13(10), 714-734.

Brown, E. \& Cairns, P. (2004). A grounded investigation of game immersion. In Proceedings of the conference on human factors in computing systems (CHI 2004) (pp. 1279-1300). New York: ACM Press

Castronova, E. (2005). Synthetic worlds. Chicago: The University of Chicago Press.

Deleuze, G. (1990). Bergonism. (H. Tomlinson \& B. Habberjam, Trans). New York: Zone Books (1988).

Dionisio, J. D. N., Burns, W. G., \& Gilbert, R. (2013). 3D Virtual worlds and the metaverse: Current status and future possibilities. ACM Computing Surveys (CSUR), 45(3), 34.

Duncan, I., Miller, A., \& Jiang, S. (2012). A taxonomy of virtual worlds usage in education. British Journal of Educational Technology, 43(6), 949-964.

Fisher, E. (2010). Contemporary technology discourse and the legitimation of capitalism. European Journal of Social Theory, 13(2), 229-252.

Gee, J. P. (2003). What video games have to teach us about learning literacy. New York: Palgrave Macmillan.

Ghanbarzadeh, R., Ghapanchi, A. H., Blumenstein, M., \& Talaei-Khoei, A. (2014). A decade of research on the use of three-dimensional virtual worlds in health care: A systematic literature review. Journal of medical Internet Research, 16(2), e47. 
Grassian, E., \& Trueman, R. B. (2007). Stumbling, bumbling, teleporting and flying... librarian avatars in Second Life. Reference Services Review, 35(1), 84-89.

Grenfell, J., \& Warren, I. (2010). Virtual worlds to enhance student engagement. The International Journal of Technology, Knowledge and Society, 6(1), 25-40.

Honey, M., Connor, K., Veltman, M., Bodily, D., \& Diener, S. (2012). Teaching with Second Life: Hemorrhage management as an example of a process for developing simulations for multiuser virtual environments. Clinical Simulation in Nursing, 8(3), 79-85.

Jarmon, L., \& Sanchez, J. (2008). The Educators Coop: A virtual world model for real world collaboration. Proceedings of the American Society for Information Science and Technology, 45, 1-10.

Johnson, L., \& Levine, A. H. (2008). Virtual worlds: Inherently immersive, highly social learning spaces. Theory Into Practice, 47(2), 161-170.

Kemp, J. W., Livingstone, D., \& Bloomfield, P. R. (2009). SLOODLE: Connecting VLE tools with emergent teaching practice in Second Life. British Journal of Educational Technology, 40(3), 551-555.

Lehdonvirta, V. (2010). Virtual worlds don't exist: Questioning the dichotomous approach in MMO studies. The International Journal of Computer Game Research, 10(1)

McArdle, G., \& Bertolotto, M. (2012). Assessing the application of three-dimensional collaborative technologies within an e-learning environment. Interactive Learning Environments, 20(1), 57-75.

Minocha, S., Tran, M., \& Reeves, A. (2010). Conducting empirical research in virtual worlds: Experiences from two projects in Second Life. Journal of Virtual Worlds Research, 3(1), 3-21.

Salen, K., \& Zimmerman, E. (2004). Rules of play—game design fundamentals. Cambridge, MA: The MIT Press.

Schroeder, R. (2008). Defining virtual worlds and virtual environments. Journal of Virtual Worlds Rresearch, $1(1)$

Sclater, M., \& Lally, V. (2013). Virtual voices: Exploring creative practices to support life skills development among young people working in a virtual world community. International Journal of Art and Design Education, 32(3), 331-344.

Shields, R. (2003). The virtual. New York: Routledge.

Singh, N., \& Lee, M. J. (2009). Exploring perceptions toward education in 3-D virtual environments: An introduction to "Second Life". Journal of Teaching in Travel \& Tourism, 8(4), 315-327.

Stone, J. (2013). Immersive virtual reality, presence and engagement: What is the pedagogic value of immersive virtual worlds?. In EC-TEL Doctoral Consortium (pp. 115-122).

Webber, S. (2013). Blended information behaviour in Second Life. Journal of Information Science, 39(1), 85-100.

Yee, N., Bailenson, J. N., Urbanek, M., Chang, F., \& Merget, D. (2007). The unbearable likeness of being digital: The persistence of nonverbal social norms in online virtual environments. CyberPsychology \& Behavior, 10(1), 115-121.

Carina Girvan is a lecturer in Education in the School of Social Sciences at Cardiff University. Her research has focused on the pedagogical underpinning of learning experiences in virtual worlds. 